\title{
Analysis of Rectangular and Circular Cross-section Power Hardening Elements Under Pure Bending
}

\author{
Serge jus Rimovskis*, Arturas Sabaliaus kas \\ Department of Mechanical Engineering, Siauliai University, Siau liai, LT-76353, Lithuania
}

\begin{abstract}
Eng ineering theory of elastic plastic bending is used in solving a grate variety of strength and durability problems. The accuracy of analysis depends on stress-strain curve idealizat ion model. The elastic linear hardening stress-strain relation is simple and frequently used. But the actual stress-strain behaviour of ductile material in plastic reg ion is non-linear and, therefore, elastic power hardening material model is more preferable. Moreover, the properties of monotonic tension and compression as proportional limits and parameters of hardening can differ. In this regard, analytical solution for rectangular and circular cross-section elements loading by monotonic elastic plastic pure bending is presented in the paper. The simple power relation of stress and strain response is used. The equations describing deviation of the stress neutral axis from centroidal axis of an element and dimensionless bending mo ment are derived.
\end{abstract}

Keywords Pure Bending, Elastic Plastic, Power Hardening

\section{Introduction}

The study of elastic-plastic bending has a wide field of application. Engineering and mathematical theories of plastic bending are well studied in T. X. Yu at al. book[1]. The authors include many experimental verifications as well as theoretical results. Elastic-plastic analysis of beam-like structures of different cross-sections under bending is considered in[2-10]. The authors employ different models of non-linear stress-strain idealization. The most used models are linear and power hardening. M. Daunys performed theoretical analysis of rectangular cross-section linear hardening beam subjected to static and cyclic pure bending[11]. The author analyses the drift of the stress neutral axis from the centroidal axis of beam, if parameters of plastic tension and compression (proportional limits, modulus of hardening) are not equal. Circular cross-section linear hardening element under pure bending is studied in [12, 13]. Derived equations are fitted to analysis of cyclic pure bending.

Power hardening stress-strain curve model is more preferable in analytical researches of strength of structures than linear hardening one because of better accuracy. Therefore, using of such a kind of idealization in pure bending analysis has not only theoretical but practical importance and applications as well.

* Corresponding author:

s.rimovskis@tf.su.lt (Sergejus Rimovskis)

Published online at http://journal.sapub.org/ijme

Copyright (C) 2012 Scientific \& Academic Publishing. All Rights Reserved

\section{Pure Bending of Rectangular Cross-Section Element}

It is assumed that in case of pure bending a transverse plane section perpendicular to the centroidal axis of the beam before deformation remains plane after deformation and that a cross-section is perpendicular to the centroidal axis after bending. It is also assumed that normal stresses and strains in the transverse and lateral directions are neglected and every fiber of the beam is either under uniaxial tension or under uniaxial co mpression. Therefore stress and strain distribution in the longitudinal cross-section of an element fit the schemes presented in Figs 1 and 2.

Stress $\sigma$ and strain $e$ of longitudinal fibers are expressed in dimensionless units $\bar{\sigma}=\sigma / \sigma_{p r}$ and $\bar{e}=e / e_{p r}$, where

$\sigma_{p r}$ and $e_{p r}$ are the values corresponding to proportional limit. Monotonic stress-strain characteristics (tension or compression) with lower rate of proportional limit are marked by index 1. Therefore, $e_{p r 2}>e_{p r 1}$ and $\sigma_{p r 2}>\sigma_{p r 1}$. Modulus of elasticity $E$ is the same for both cases. According to the law of equilibrium, we obtain

$$
\int_{-y}^{+y} \sigma b d y=0
$$

and

$$
M=\int_{-y}^{+y} \sigma b y d y,
$$


where $b$ is a width of an element and $y$ is a distance from the fiber under analys is to the centroidal axis of an element.

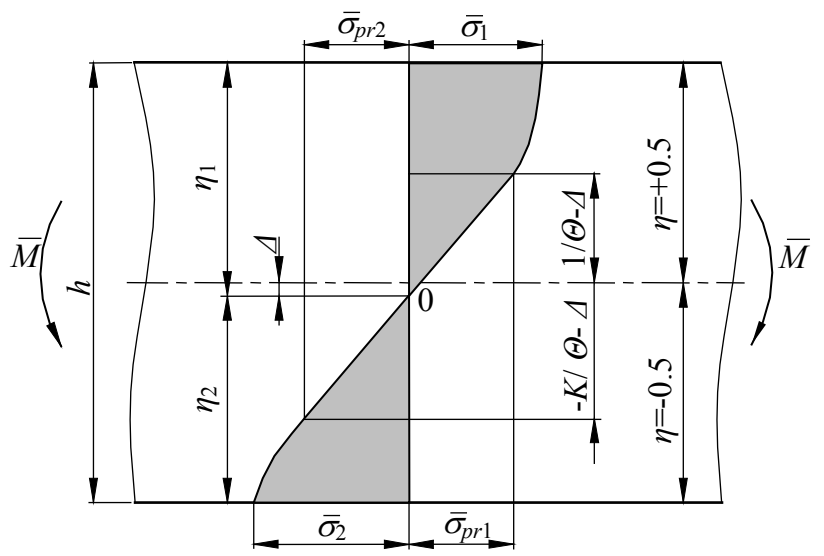

Figure 1. Stress distribution in the longitudinal cross-section of an element

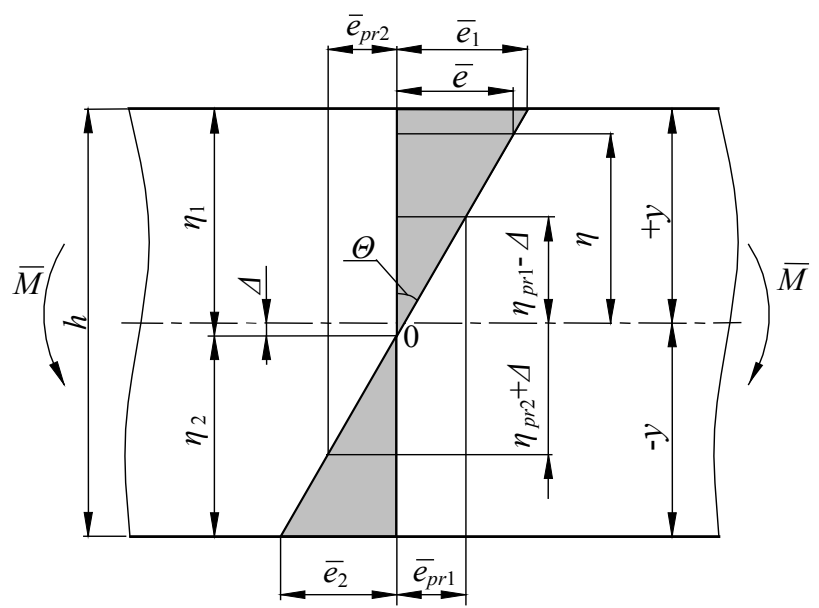

Figure 2. Strain distribution in the longitudinal cross-section of an element

According to Fig. 2, $\eta=y / h$. Hence, dimensional expression of distant $y$ is $y=h \eta$ and $d y=h d \eta$. Substituting $y$ and $d y$ in Eq. (1) we get

$$
\sigma_{p r 1} b h \int_{-0.5}^{+0.5} \bar{\sigma} d \eta=0 .
$$

It is evident that $\sigma_{p r 1} b h \neq 0$ and so Eq. (3) can be simp lified to

$$
\int_{-0.5}^{+0.5} \bar{\sigma} d \eta=0
$$

In the plastically deformed fibers on the convex side of the beam, as in case of the monotonic tension, stress-strain curve with proportional limit $e_{p r 1}$ is used, so

$$
\sigma_{1}=\sigma_{p r}\left(\frac{e}{e_{p r 1}}\right)^{m_{1}}
$$

In the fibers on the concave side of the beam with proportional limit $e_{p r 2}$ we get

$$
\sigma_{2}=\sigma_{p r 2}\left(\frac{e}{e_{p r 2}}\right)^{m_{2}}
$$

In Eq. (5) and (6) $m_{1}$ and $m_{2}$ are strain hardening exponents. After dividing Eqs. (5) and (6) by $\sigma_{p r 1}$ and rearranging, we obtain dimensionless stress expressions

$$
\bar{\sigma}_{1}=\bar{e}^{m_{1}}
$$

and

$$
\bar{\sigma}_{2}=K\left(\frac{\bar{e}}{K}\right)^{m_{2}} .
$$

In Eq. (8), $K$ is a coefficient accounting for difference of proportional limits

$$
K=\frac{e_{p r 2}}{e_{p r 1}}=\frac{\sigma_{p r 2}}{\sigma_{p r 1}} .
$$

If $K=1$ and $m_{1}=m_{2}$, the position of the neutral axis coincides with the cross-section centroidal axis. If $K>1$ and (or) $m_{1} \neq m_{2}$, the position of the neutral axis deviates from centroidal axis of an element by value $\Delta$. We mark the strain of fiber of centroidal axis as $\bar{e}_{\Delta}$ and transform Eqs. (7) and (8) to

$$
\bar{\sigma}_{1}=\left(\bar{e}+\bar{e}_{\Delta}\right)^{m_{1}}
$$

and

$$
\bar{\sigma}_{2}=K\left(\frac{\bar{e}+\bar{e}_{\Delta}}{K}\right)^{m_{2}} .
$$

It is evident, that if $\bar{e}_{\Delta} \neq 0$, the strains on the convex and concave sides of an element are not equal $\left(\bar{e}_{1} \neq \bar{e}_{2}\right)$. According to Fig. $1, \bar{e}=\Theta \eta$ and $\bar{e}_{\Delta}=\Theta \Delta$, where $\Theta$ is the dimensionless angle of cross-sections deflection. In regard to this, final expressions of plastic stress are

$$
\bar{\sigma}_{1}=(\Theta(\eta+\Delta))^{m_{1}}
$$

and

$$
\bar{\sigma}_{2}=K\left(\frac{\Theta}{K}(\eta+\Delta)\right)^{m_{2}} .
$$

In the fibers deformed elastically

$$
\bar{\sigma}=\Theta(\eta+\Delta)
$$

Hence, integral (4) can be written as a sum of three integrals representing three different reg ions of strains

$$
\begin{aligned}
& \int_{\frac{1}{\Theta}-\Delta}^{0.5}(\Theta(\eta+\Delta)) d \eta+\int_{-\frac{K}{\Theta}-\Delta}^{m_{1}} \Theta(\eta+\Delta) d \eta- \\
& \int_{\frac{K}{\Theta}+\Delta}^{\frac{1}{\Theta}-\Delta} K\left(\frac{\Theta}{K}(\eta-\Delta)\right)^{m_{2}} d \eta=0
\end{aligned}
$$

In case of pure bending, if $1<\bar{e}_{2}<K$ (the fibers on the convex side of an element exceeded $e_{p r 1}$ and the fibers on 
the concave side of an element are still under elastic conditions), Eq. (14) can by simplified

$$
\int_{\frac{1}{\Theta}-\Delta}^{0.5}(\Theta(\eta+\Delta)) d \eta+\int_{-0.5}^{m_{1}} \Theta(\eta+\Delta) d \eta=0 .
$$

Dimensionless distant from extreme fiber with $+\sigma_{1}$ to the neutral axis is

$$
\eta_{1}=0.5-\Delta .
$$

By referring to integrals (15), (16) and Eq. (17), distant $\eta_{1}$ is found for the angle $\Theta$. Subsequently the strains $\bar{e}_{1}$ and $\bar{e}_{2}$ can be obtained.

The integral (2) can be transformed considering that $\sigma=\sigma_{p r 1} \bar{\sigma}, y=h \eta$ and $d y=h d \eta$

$$
M=\int_{-0.5}^{0.5} \sigma_{p r 1} \bar{\sigma} \eta b h^{2} d \eta,
$$

or

$$
M=\sigma_{p r} \frac{b h^{2}}{6} 6 \int_{-0.5}^{0.5} \bar{\sigma} \eta d \eta .
$$

The pure bending moment corresponding to the proportional limit stress is

$$
M_{p r}=\sigma_{p r 1} W=\sigma_{p r 1} \frac{b h^{2}}{6} .
$$

So, the integral (19) can be written in dimensionless form

$$
\bar{M}=\frac{M}{M_{p r}}=6 \int_{-0.5}^{0.5} \bar{\sigma} \eta d \eta .
$$

Substituting $\bar{\sigma}$ (Eqs. (12)-(14)) in (21) we get:

$$
\begin{aligned}
& \bar{M}=6\left\{\int_{\frac{1}{\Theta}-\Delta}^{0.5}(\Theta(\eta+\Delta)) \eta d \eta+\int_{-\frac{K}{\Theta}-\Delta}^{m_{1}} \Theta(\eta+\Delta) \eta d \eta+\right. \\
& \left.+\int_{\frac{K}{\Theta}+\Delta}^{0.5} K\left(\frac{\Theta}{K}(\eta-\Delta)\right)^{m_{2}} \eta d \eta\right\}
\end{aligned}
$$

In case, if $1<\bar{e}_{2}<K$

$$
\left.\bar{M}=6\left\{\int_{\frac{1}{\Theta}-\Delta}^{0.5}(\Theta(\eta+\Delta)) \eta\right)_{1}^{m_{1}} \eta \int_{-0.5}^{\frac{1}{\Theta}-\Delta} \Theta(\eta+\Delta) \eta d \eta\right\} .
$$

Integrals (21) and (22) allow to determine moment $\bar{M}$ depending on maximum strain. Analytical expressions of integrals (15), (16), (22) and (23) exist, but are not presented.

Figs. 3 and 4 represent curves of functions $\eta_{1}=f(\bar{e})$ and $\bar{M}=f(\bar{e})$ in the strain range $\bar{e}=1-9$ for the fallowing values of parameters $K$ and $m: K=1.0 ; K=1.1 ; m=0.1$; $m=0.2 ; m=0.3 ; m=0.4$.

\section{Pure Bending of Circular Cross-Section Element}

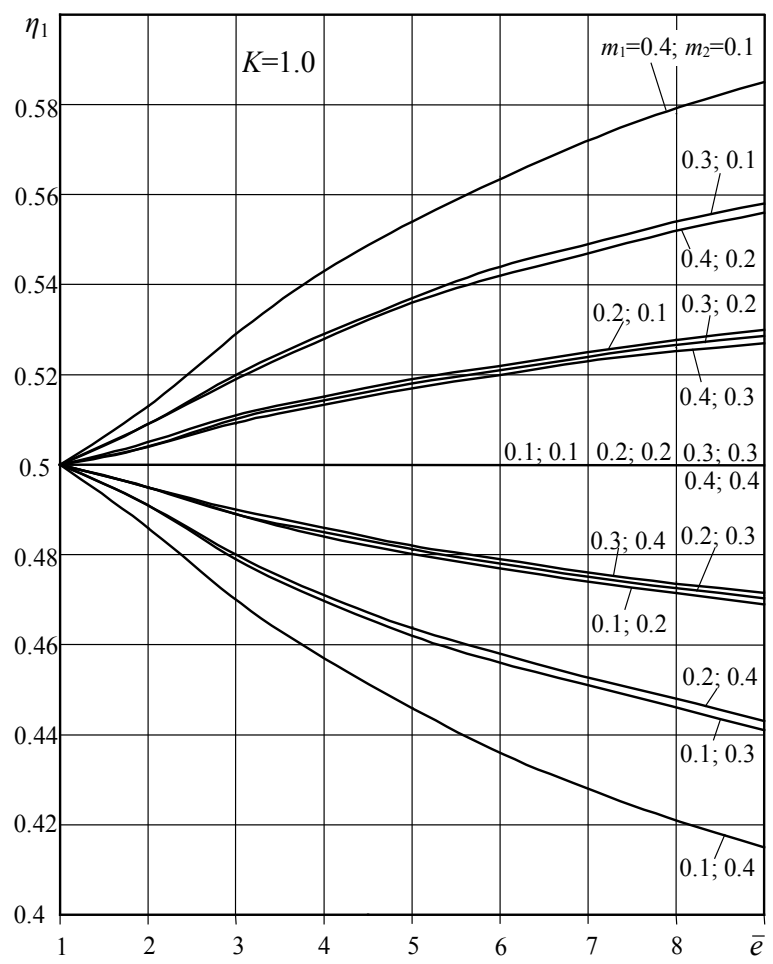

a)

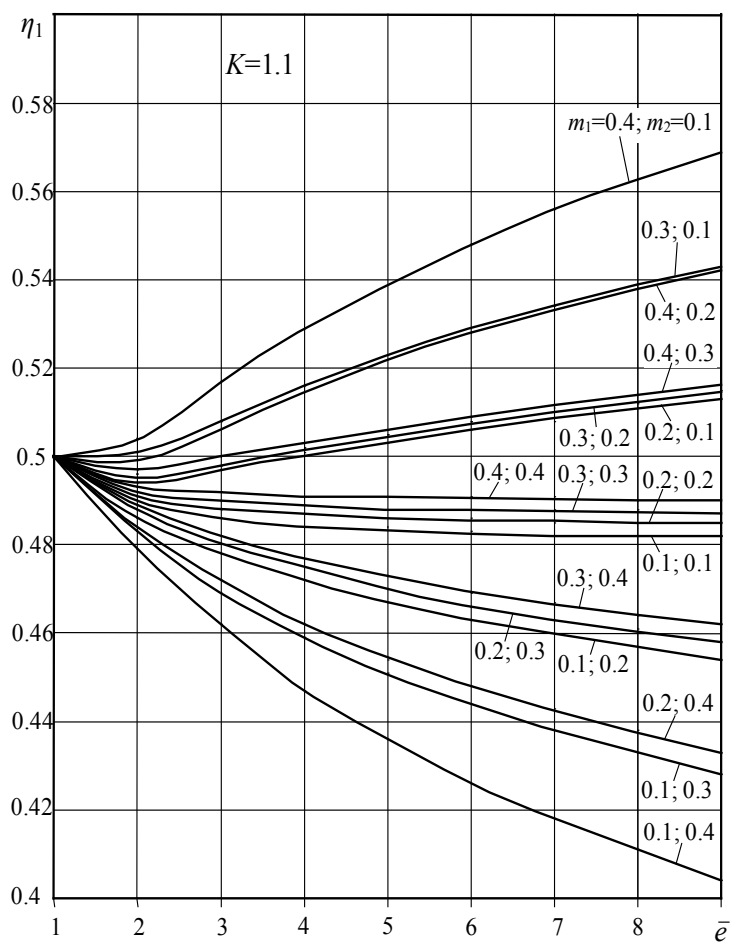

b)

Figure 3. The distance from extreme fiber with $+\sigma_{1}$ to the neutral axis of rectangular cross-section element vs. monotonic tension strain, for $K=1.0$ (a) and $K=1.1$ (b) 
In case of elastic plastic pure bending of circular cross-section beam, the stress and strain distribution in the longitudinal cross-section also fit the schemes presented in Figs. 1 and 2. But in the transversal cross-section some differences are seen (see Fig. 5).

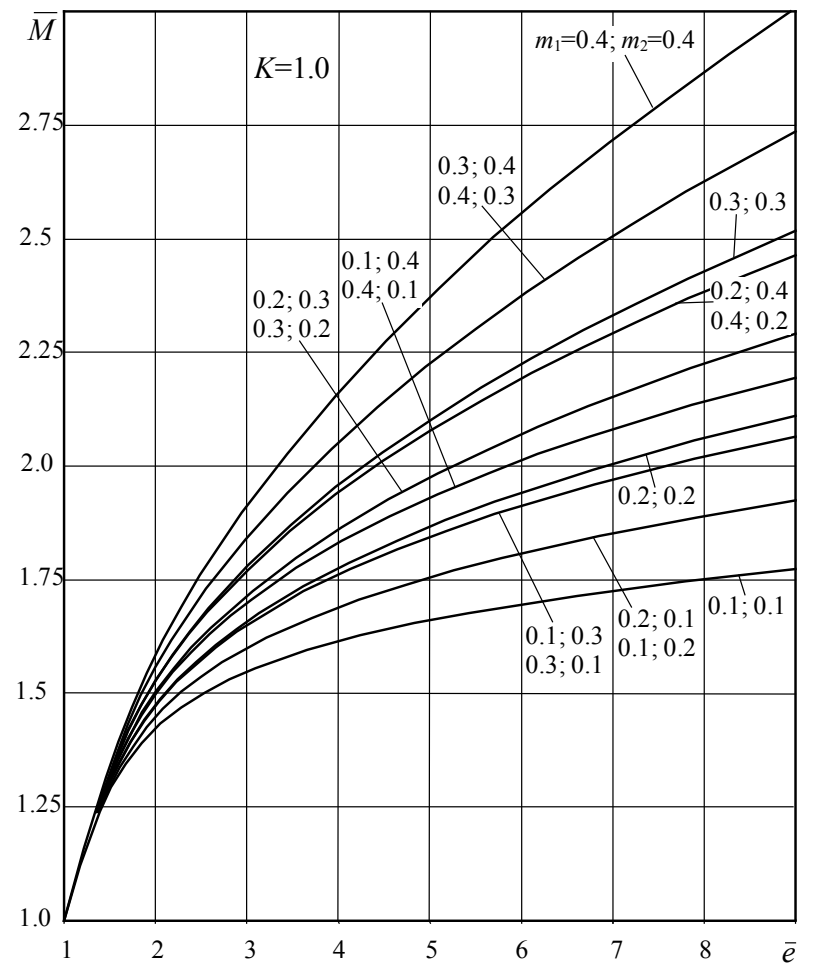

a)

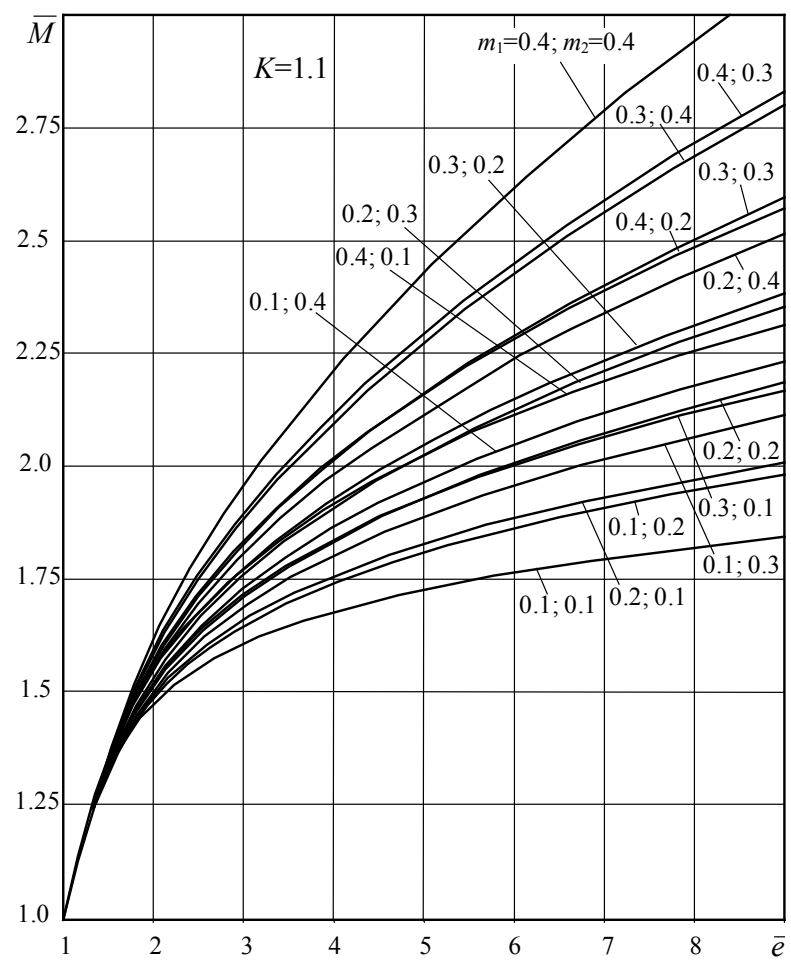

b)

Figure 4. Dimensionless bending moment of rectangular cross-section element vs. monotonic tension strain, for $K=1.0$ (a) and $K=1.1$ (b)

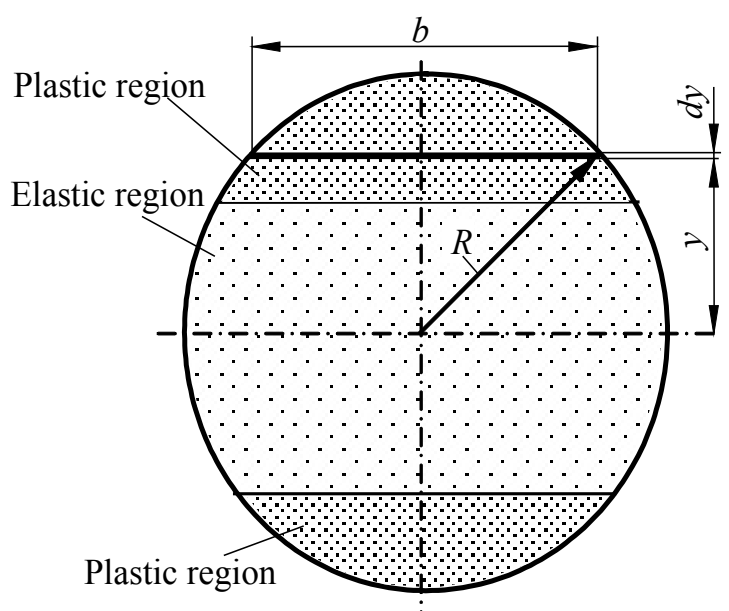

Figure 5. The regions of elastic and plastic strains in the transversal cross-section of a circular element

The width of circular cross-section element $b$ depends on distance $y$. Considering, that $h=2 R$ and $y=2 R \eta$ we get

$$
b=2 R \sqrt{1-4 \eta^{2}} .
$$

It is evident, that $d y=2 R d \eta$, so integral (1) can be expressed as

$$
4 \sigma_{p r 1} R^{2} \int_{-0.5}^{+0.5} \bar{\sigma} \sqrt{1-4 \eta^{2}} d \eta=0
$$

and simplified to

$$
\int_{-0.5}^{+0.5} \bar{\sigma} \sqrt{1-4 \eta^{2}} d \eta=0
$$

Using $\bar{\sigma}$ defined by Eqs. (12)-(14), allows to transform integral (26)

$$
\begin{aligned}
& \int_{\frac{1}{\Theta}-\Delta}^{0.5}(\Theta(\eta+\Delta)) \sqrt{1-4 \eta^{2}} d \eta+ \\
& +\int_{\frac{K}{\Theta}-\Delta}^{m_{1}} \Theta(\eta+\Delta) \sqrt{1-4 \eta^{2}} d \eta- \\
& -\int_{\frac{K}{\Theta}+\Delta}^{\frac{1}{\Theta}-\Delta} K\left(\frac{\Theta}{K}(\eta-\Delta)\right)^{m_{2}} \sqrt{1-4 \eta^{2}} d \eta=0
\end{aligned}
$$

And in case, if $1<\bar{e}_{2}<K$

$$
\begin{aligned}
& \int_{\frac{1}{\Theta}-\Delta}^{0.5}(\Theta(\eta+\Delta)) \sqrt{1-4 \eta^{2}} d \eta+ \\
& +\int_{-0.5}^{m_{1}} \Theta(\eta+\Delta) \sqrt{1-4 \eta^{2}} d \eta=0
\end{aligned}
$$

Next we transform the integral (2) to

$$
M=\int_{-0,5}^{0,5} 2 \bar{\sigma} \sigma_{p r 1} 2 R \sqrt{R^{2}-4 R^{2} \eta^{2}} d \eta
$$


and

$$
M=\frac{\sigma_{p r 1}(2 R)^{3} 32 \pi}{32 \pi} \int_{-0,5}^{0,5} \bar{\sigma} \eta \sqrt{1-4 \eta^{2}} d \eta .
$$

In case of pure bending of circular cross-section element, the bending mo ment corresponding to the proportional limit is

$$
M_{p r 1}=\sigma_{p r 1} W=\frac{\sigma_{p r 1}(2 R)^{3} \pi}{32} .
$$

Hence, dimensionless bending moment

$$
\bar{M}=\frac{M}{M_{p r 1}}=\frac{32}{\pi} \int_{-0.5}^{0.5} \bar{\sigma} \eta \sqrt{1-4 \eta^{2}} d \eta .
$$

The final expression of $\bar{M}$ is

$$
\begin{gathered}
\bar{M}=\frac{32}{\pi}\left\{\int_{\frac{1}{\Theta}-\Delta}^{0.5}(\Theta(\eta+\Delta)) \sqrt{1-4 \eta^{2}} \eta d \eta+\right. \\
+\int_{\frac{K}{\Theta}-\Delta}^{m_{1}} \Theta(\eta+\Delta) \sqrt{1-4 \eta^{2}} \eta d \eta+ \\
\left.\int_{\frac{K}{\Theta}+\Delta}^{0.5} K\left(\frac{\Theta}{K}(\eta-\Delta)\right)^{m_{2}} \sqrt{1-4 \eta^{2}} \eta d \eta\right\}
\end{gathered}
$$

In case, if $1<\bar{e}_{2}<K$

$$
\begin{aligned}
\bar{M}=\frac{32}{\pi} & \left\{\int_{\frac{1}{\Theta}-\Delta}^{0.5}(\Theta(\eta+\Delta)) \sqrt{1-4 \eta^{2}} \eta d \eta+\right. \\
& \left.+\int_{-0.5}^{m_{1}} \Theta(\eta+\Delta) \sqrt{1-4 \eta^{2}} \eta d \eta\right\}
\end{aligned}
$$

Analytical expressions of integrals (27), (28), (33) and (34) do not exist. These integrals can be solved numerically.

Theoretical $\eta_{1}=f(\bar{e})$ and $\bar{M}=f(\bar{e})$ curves for the same values of $K$ and $m$ are presented in Figs. 6 and 7 .

Mechanical properties of majority of ductile materials determined by tensile and compressive testing usually differ. Consequently constants of idealized stress-strain curves (proportional limits and parameters of hardening) are also different. Analysis of pure bending of rectangular and circular cross-section element can be realized with regard to this. The values of dimensionless bending moment can be found directly from presented diagrams or calculated by deduced formulas.

The developed analytical solution of monotonic elastic plastic pure bending can be used to analyse the cyclic elastic plastic pure bending as it is presented by formulas $[11,12]$.

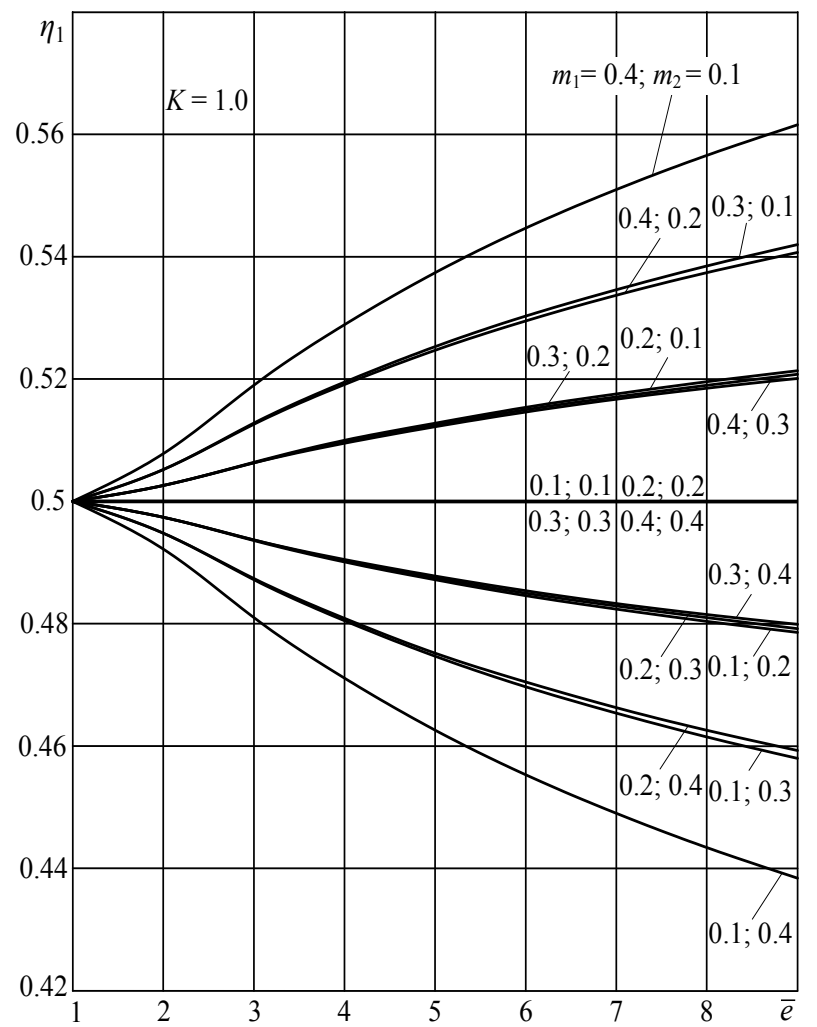

a)

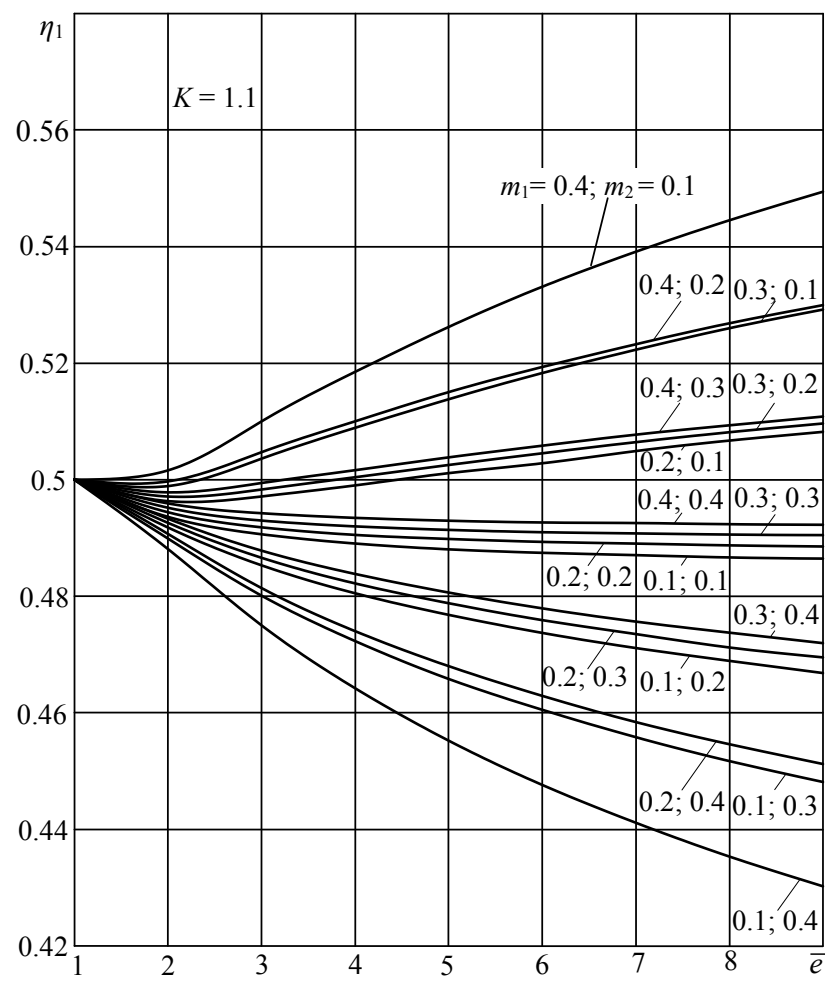

b)

Figure 6. The distance from extreme fiber with $+\sigma_{1}$ to the neutral axis of circular cross-section element vs. monotonic tension strain, for $K=1.0$ (a) and $K=1.1$ (b) 


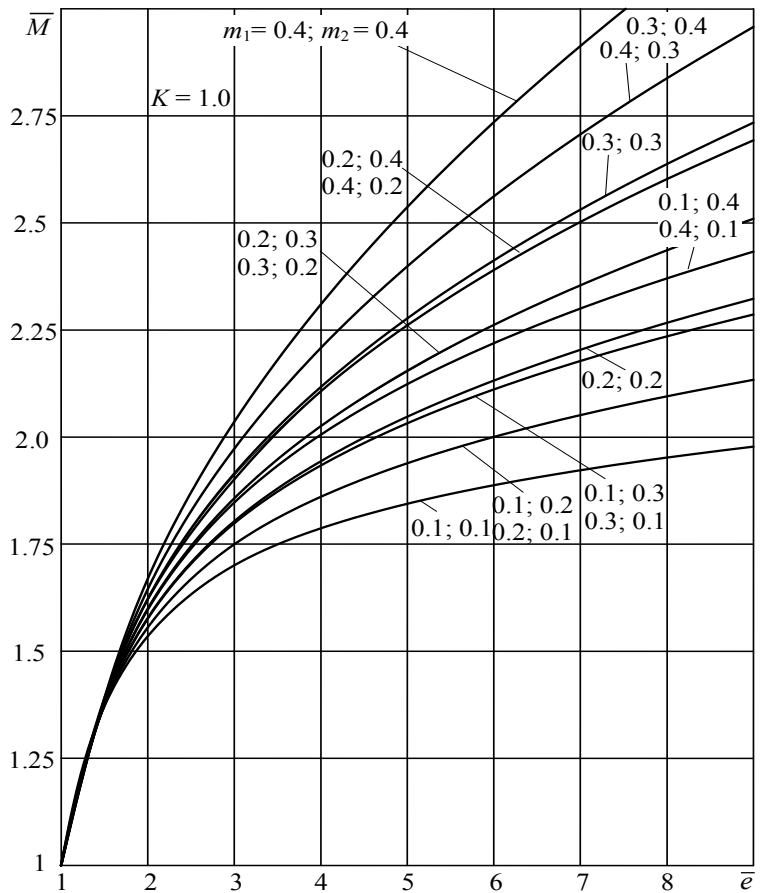

a)

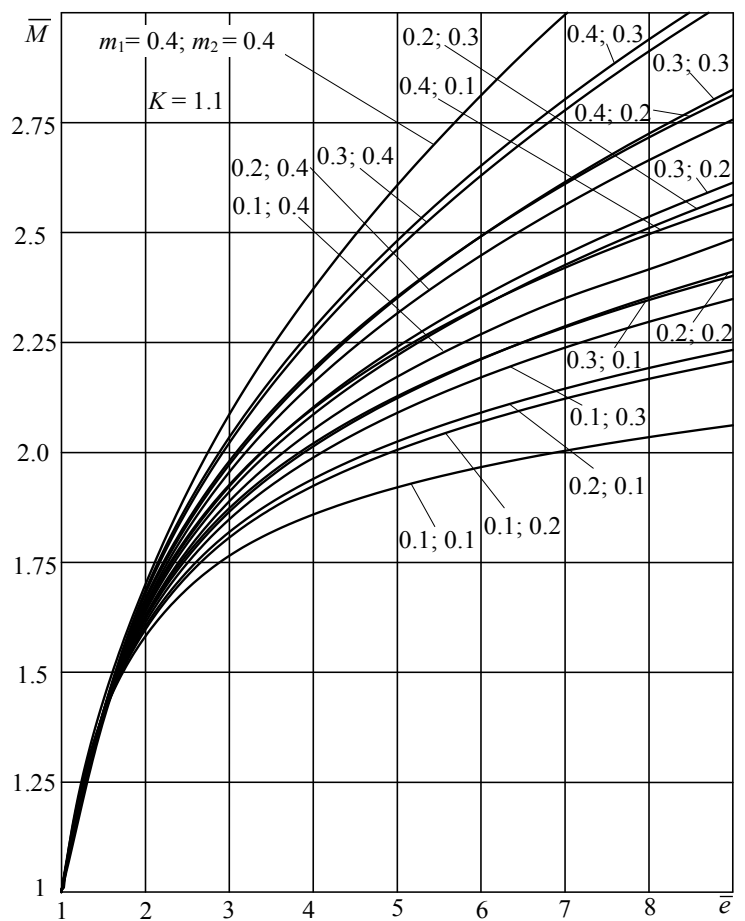

b)

Figure 7. Dimensionless bending moment of circular cross-section element vs. monotonic tension strain, for $K=1.0$ (a) and $K=1.1$ (b)

\section{Conclusions}

Elastic-power hardening idealization of uniaxial stress strain curve is used in theoretical research of rectangular and circular cross-section element under elastic plastic pure bending. Presented derivations allow to calculate deviation of stress neutral axis from centroidal axis of an element and pure bending mo ment versus monotonic strain if parameters of plastic tension and compression (proportional limits, exponents of hardening) are not equal. Derived expressions of bending moment can be used in theoretical research of cyclic elastic plastic pure bending.

\section{REFERENCES}

[1] Yu T. X., Zhang L. C. Plastic Bending. Theory and Applications. Series on En gineering Mechanics, vol. 2, World Scientific. 572 pp. 1996.

[2] Štok B., Halilovič M.. Analytical Tracing of the Evolution of the Elasto-Plastic State during the Bending of Beams with a Rectangular Cross-Section. Journal of Mechanical Engineering, vol. 53, p. 806-818. 2007.

[3] Štok B., Halilovič M. Analytical Solutions in Elasto-Plastic Bending of Beams with Rectangular Cross-Section. Applied Mathematical Modeling, vol. 33, p. 1749-1760. 2009.

[4] Rees D. W. Descriptions of Reversed Yielding in Bending. Journal of Mechanical Engineering Science, vol. 221, p. 981-991. 2007.

[5] Bin J., Wanji C. A New Analytical Solution of Pure Bending Beam in Couple Stress Elasto-Plasticity: Theory and Applications. International Journal of Solids and Structures, vol. 47, p. 779-785. 2010.

[6] Eraslan A. N., Arslan E. A Concise Analytical Treatment of Elastic-Plastic Bending of a Strain Hardening Curved Beam. Journal of Applied Mathematics and Mechanics, vol. 88, p. 600-616. 2008 .

[7] Arslan E., Eraslan A. N. Analytical solution to the bending of a nonlinearly hardening wide curved bar. Acta Mechanica, vol. 210, p. 71-84. 2010

[8] Yang J., Reid S. R. Approximate estimation of Hardening-Softening Behaviour of Circular Pipes Subjected to Pure Bending. Acta Mechanica Sinica, vol. 13, p. 227-240. 1997.

[9] Lanzagorta J. L., Meizoso A. M. 3-Point Bending of Bars and Rods Made of Materials Obeying a Ramberg-Osgood Criterion. World Journal of Mechanins, vol. 1, p. 71-77. 2011.

[10] Chakrabarty J., Lee W.B., Chan K.C. An Analysis of the Plane-Strain Bending of an Orthotropic Sheet in the Elastic/Plastic range. Journal of Materials Processing Technology, vol. 104, p. 48-52. 2000.

[11] Daunys M. Solution for Rectangular Cross-Section Bars above Proportionality Stress. Electrotechnics and Mechanics. p. 61-69. 1969 (in Lithuanian).

[12] Daunys M., Rimovskis S. Analy sis of Circular Cross-Section Bar, Loaded by Static and Cyclic Elasto-Plastic Pure Bending. Mechanika, vol. 33, p. 5-10. 2002.

[13] Daunys M., Rimovskis S. Analy sis of Circular Cross-Section Element, Loaded by Static and Cyclic Elastic-Plastic Pure Bending. International Journal of Fatigue, vol. 28, p. 211-222. 2006. 